індекс складності оптичної схеми і деякі евристичні правила формалізації переходу від вимог технічного завдання до вибору елементної бази оптичної системи.

Таким чином, показана актуальність формалізації процесу проектування оптичних систем, основами якої $€$ питання класифікації і каталогізації в зв'язку з великими архівами розрахованих оптичних систем. При цьому класифікаційний підхід до каталогів оптичних систем може бути основним фактором скорочення термінів нових розробок. Такий підхід може використовуватися для розуміння необхідності створення архівів розробок і класифікації цієї інформації.

.Ключові слова: класифікація, каталог, оптотехніка, розрахунок оптичних схем, оптимізація, відомості про основні параметри системи, якість зображення.

\author{
N. K. Artioukhina, A. P. Shkadarevich \\ ${ }^{1)}$ Belarussian National Technical University. Minsk, Republic Belarus; \\ ${ }^{2)}$ Scientific-technical center «LEMT», BelOMO, Minsk, Belarus
}

\title{
CLASSIFICATION APPROACHES FOR THE SOLUTION OF THE TASK OF THE CHOICE OF BASIC MODEL OF OPTICAL SYSTEMS
}

The article shows the urgency of creating libraries of computer programs that are designed for modeling and technological adaptation of optical systems of any class, taking into account the specifics of design developments, the features of technological processes for manufacturing optical parts, and assembling ready units and devices. This is due to the need to computerize the design and processing of measurement results for optical system parameters. One of the trends in computational optics is the constant search for new circuits with improved characteristics.

Thus, the main objective of the work is to create a classification approach to the choice of the initial system (the starting point of the OS design).

Classification bases of synthesis of optical systems are presented: a classification of optical schemes, modules, elements; their types; a cataloguing optical systems; the method of formalization of knowledge by means of decisions tables; an index of complexity of the optical scheme and some heuristic rules of formalization of transition from requirements of the preliminary specification to a choice of the element base of optical systems.

Thus, the relevance of the formalization of the OS design process is shown, the bases of which are classification and cataloging issues in connection with large archives of calculated OS. At the same time, the classification approach to the catalogs of optical systems may be the main factor in reducing the time for new developments. This approach can be used to understand the need to create development archives and classify this information.

Keywords: classification, catalog, optics engineering, calculation of optical schemes, optimization, report of key parameters of system, quality of the image.

Надійшла до редакиіі 12 березня 2018 року

Речензовано

27 березня 2018 року

\section{УДК 621: 681.7 \\ ОПТИКО-ЭЛЕКТРОННЫЙ ДЕТЕКТОР ДОРОЖНОЙ РАЗМЕТКИ}

\author{
Стадничук В. С., Кондратенко Д. Ю. \\ Начиональный технический университет Украины «Киевский политехнический инсти- \\ тут имени Игоря Сикорского», Киев, Украина \\ E-mail: SVS97@,i.ua, deka@email.ua
}

\begin{abstract}
В данной работе рассмотрены и проанализированы существующие конструкции оптико-электронных систем активной безопасности, помощи вождению, основные причины возникновения ДТП, кончепџии автономного вождения, предложена разработка собственного универсального оптико-электронного прибора для всех типов автомобилей. Основным недостатком таких систем является отсутствие мобильности. Анализ существующих систем активной безопасности выявил ряд бесспорных преимуществ оптикоэлектронных систем, а также доказал эффективность их применения.
\end{abstract}

Ключевые слова: ADAS, Autonomous Driving Car, SLAM, LIDAR, SAE, обработка изображений. 


\section{Введение}

В настоящее время компьютеризация в автомобилестроении развивается быстрыми темпами и играет огромную роль в безопасности дорожного движения. При помощи компьютерных технологий автоматизируется широкий круг процессов, которые в недалеком прошлом возлагались на человека.

Решение проблемы распознавания и обработки изображений и, как следствие, предотвращение аварийной ситуации является довольно важным аспектом безопасности и контроля дорожной ситуации. Системы активной безопасности позволяют корректировать ошибочные действия водителя, избегать экстренных ситуаций, повысить безопасность дорожного движения.

Актуальной, востребованной и интересной темой этого исследования является анализ и разработка собственного универсального прибора «консультации» водителя (ADAS) в вопросе активной безопасности.
Данные исследования (NMVCCS) [1] показали, что около 95\% дорожно-транспортных происшествий (ДТП) были вызваны ошибкой водителя. Из них:

$>$ 27,7\% ДТП были вызваны действиями водителя (например, отсутствие контроля направления, превышение скоростного режима);

$>25,4 \%$ ДТП вызваны ошибочными действиями водителя в экстренной ситуации (например, резкое торможение на скользкой дороге);

$>22,5 \%$ ДТП вызваны критическим состоянием водителя (сонливость, физические нарушения);

$>\quad 19,8 \%$ ДТП вызваны невнимательностью водителя (например, отвлечение на телефонный звонок).

Данная тема является актуальной, поскольку более $40 \%$ ДТП происходит из-за невнимательности водителя (рис. 1), а электронные ассистенты сводят этот процент к минимуму [2].

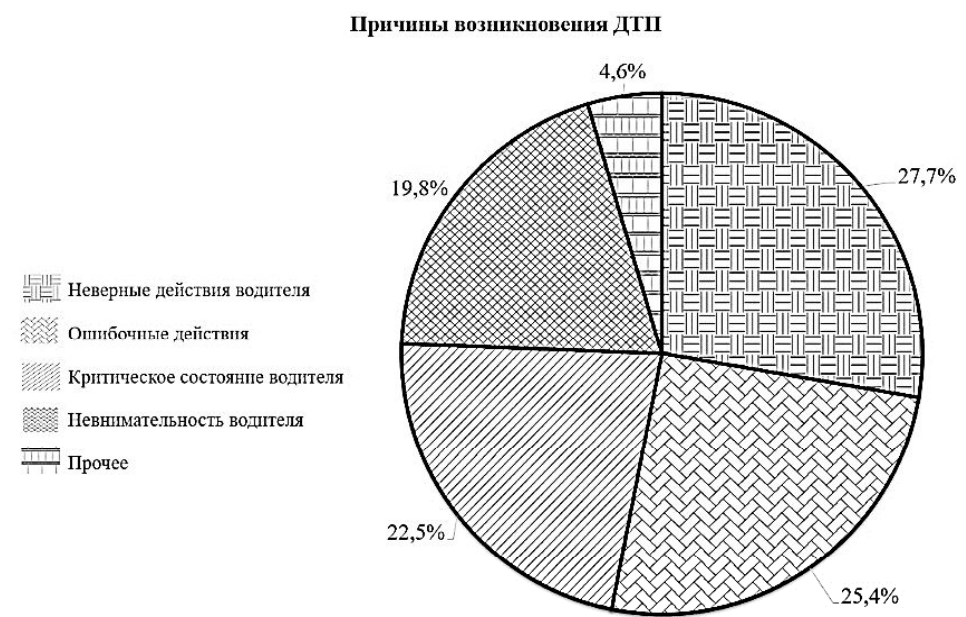

Рис. 1. Диаграмма причин возникновения ДТП [1]

Существующие оптические системы активной безопасности

На сегодняшний день большинство автопроизводителей применяют оптические активные системы безопасности, как наиболее информативные. В паре с оптическими применяются системы, построенные на ультразвуковых датчиках, однако одиночное применение данных систем мало эффективно. В рамках данного исследования рассмотрены оптические системы активной безопасности [3].

В своей основе данные системы содержат оптический приёмник излучения (в большинстве случаев, видеокамера), микропроцессорный блок, устройство вывода информации/воздействия на органы управления. Требования к таким приёмникам: приемлемое изображение в дневных и ночных условиях, минимальная инерционность, большой угол обзора, минимальные аберрации, малая масса и габариты.
Все системы активной безопасности можно разделить на две большие группы: ADAS и Autonomous Driving Car (рис. 2).

\section{Коншепиия ADAS}

Расширенная система помощи водителю («Advanced driver assistance systems», ADAS) - это системы, которые помогают водителю в процессе движения. Они должны повысить безопасность управления автомобилем и, в целом, безопасность дорожного движения.

Все перечисленные выше системы относятся к расширенной системе помощи водителю, хотя могут использоваться для автономного вождения (рис. 3).

Данная технология обеспечивает лишь «консультирование» водителя о ситуации на дороге. Взять полный контроль ситуации на себя она не может. Поэтому водителю нельзя полагаться на электронных ассистентов [4].

Рассмотрим системы-составляющие ADAS. 


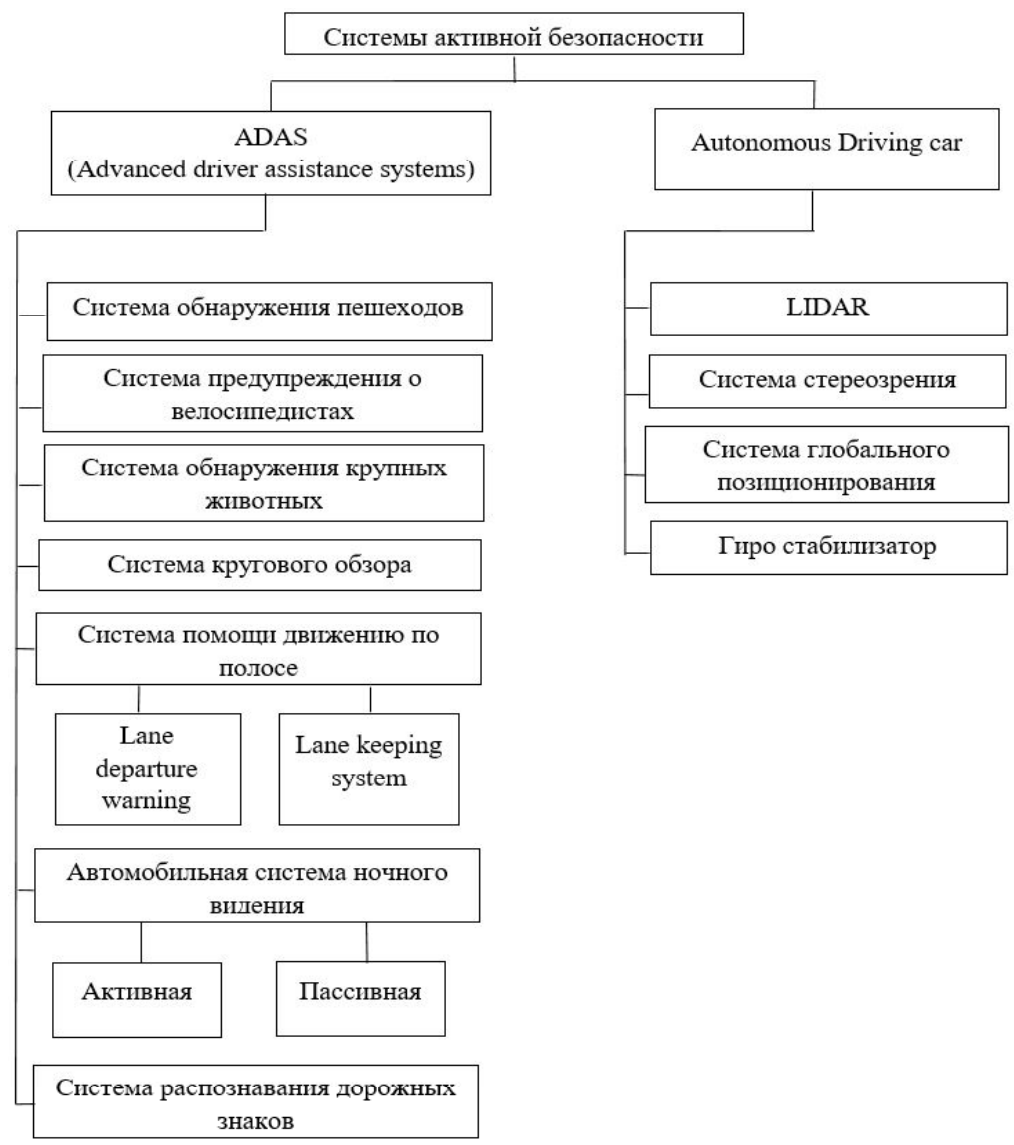

Рис. 2. Системы активной безопасности

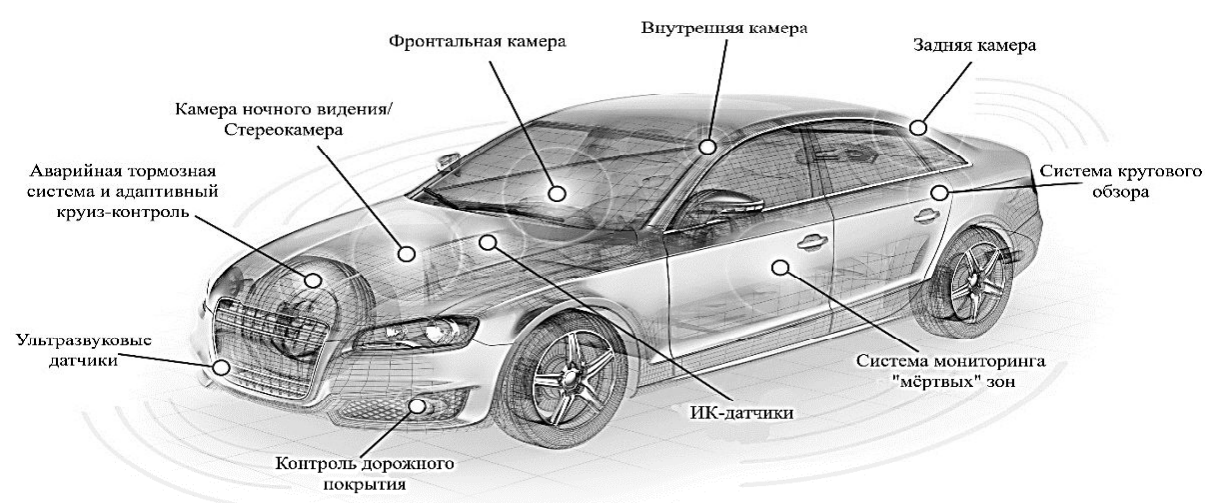

Рис. 3. Схематичное представление автомобиля с ADAS

\section{Автопилотируемые автомобили}

Также в наше время интенсивно развиваются автопилотируемые автомобили. В общем, все перечисленные выше системы плавно подводят к идее о беспилотном транспортном средстве. В своей конструкции они содержат камеры высокого разрешения, множество датчиков (лидары, ультразвуковые), высокоточный GPS-приёмник.

В данный момент автопилотируемые автомобили не в состоянии обеспечить полную автоном- ность движения, хоть в некоторых ситуациях всё же необходимо вмешательство водителя.

Autonomous Driving (Autonomous car)

Автономный автомобиль - беспилотное наземное транспортное средство, представляет собой транспортное средство, которое способно к ощущению его окружения и навигации без участия человека.

Автономные автомобили используют множество методов для обнаружения их окружения, та- 
ких как радар, лазерный луч, GPS, одометрия и компьютерное зрение. Расширенные системы управления интерпретируют информацию, полученную с датчиков для определения соответствующих путей навигации, а также препятствий и соответствующих обозначений. Автономные автомобили должны иметь системы управления, которые способны различать другие автомобили и препятствия на дороге.

В современных беспилотных автомобилях используются алгоритмы на основе Байесовского метода одновременной локализации и построения карт (SLAM, simultaneous localization and mapping). Суть работы алгоритмов состоит в комбинировании данных с датчиков автомобиля (real-time) и данных карт (offline). SLAM и метод обнаружения и отслеживания движущихся объектов (DATMO, detection and tracking of moving objects) разработаны и применяются в Google [6], [7].

Обычно устанавливаемые датчики:

- LIDAR - дальномер оптического распознавания;

- Система стереозрения;

- Система глобального позиционирования (GPS, Глонасс);

- Гиростабилизатор

Программное обеспечение беспилотного автомобиля может включать машинное зрение и нейросети [8].

В своей основе автономные автомобили содержат все системы ADAS [9].
Сравнительный анализ систем активной безопасности

Из приведенной ниже таблицы [8] видно, что наиболее востребованным устройством приёма информации являются видеокамеры и другие оптические приёмники излучения. Наиболее информативной является концепция Autonomos Driving Car, поскольку обладает полным спектром информации с разных устройств и датчиков, которые могут замещать друг друга. Например, в условиях недостаточной видимости камера, работающая в видимом диапазоне, является мало информативной - в этом случае компьютер будет получать информацию с других датчиков.

\section{Результаты исследований}

В рамках данного исследования был разработан оптико-электронный прибор - детектор дорожной разметки, который звуковым сигналом и световой индикацией предупреждает водителя о сходе со своей полосы движения.

Недостатком решений, предоставляемых на авторынке, является отсутствие универсальности, дороговизна, сложность установки, отсутствие возможности вмешательства в работу прибора. Система, разработанная концерном под один автомобиль, не подойдёт для автомобиля другого концерна. Как правило, системы подобного типа невозможно модифицировать - они обладают закрытым исходным кодом.

Таблица. Сравнение оборудования систем активной безопасности [8]

\begin{tabular}{|c|c|c|c|c|c|c|c|c|c|c|c|}
\hline Системы безопасностит & $\begin{array}{c}\text { Камера } \\
\text { фронтальная }\end{array}$ & Радары & \begin{tabular}{c|} 
Камера \\
задняя
\end{tabular} & $\begin{array}{l}\text { Камеры по } \\
\text { периметру }\end{array}$ & \begin{tabular}{|c|} 
Лазерные \\
датчики
\end{tabular} & ИК-датчики & ИК-камера & LIDAR & GPS & $\begin{array}{c}\text { Система } \\
\text { стереозрения }\end{array}$ & Гиростабилизатор \\
\hline $\begin{array}{l}\text { Система обнаружения } \\
\text { пешеходов }\end{array}$ & + & + & - & - & - & + & + & - & - & - & - \\
\hline $\begin{array}{l}\text { Система предупреждения о } \\
\text { велосипедистах }\end{array}$ & + & + & + & + & - & - & - & - & - & - & - \\
\hline $\begin{array}{l}\text { Система обнаружения крупных } \\
\text { животных }\end{array}$ & + & + & - & - & - & - & - & - & - & - & - \\
\hline Система кругового обзора & + & - & + & + & - & - & - & - & - & - & - \\
\hline $\begin{array}{l}\text { Система помощи движению по } \\
\text { полосе }\end{array}$ & + & - & & & + & + & - & - & - & - & - \\
\hline $\begin{array}{l}\text { Автомобильная система } \\
\text { ночного видения }\end{array}$ & + & - & - & - & - & - & + & - & - & - & - \\
\hline $\begin{array}{l}\text { Система распознавания } \\
\text { дорожных знаков }\end{array}$ & + & - & - & - & - & - & - & - & - & - & - \\
\hline Autonomous Driving Car & + & + & + & + & + & + & + & + & + & + & + \\
\hline
\end{tabular}

После анализа недостатков рыночных решений был предложен вариант оптико-электронного детектора дорожной разметки, в котором отсутствуют перечисленные выше недостатки. Преимущества предложенного детектора разметки вытекают из недостатков рыночных решений, а именно: дешевизна, простота установки и юстировки, открытый исходный код, что даёт возможность вносить изменения в систему.

За основу детектора взята плата Orange Pi Zero под управлением операционной системой
Ubuntu (Linux). Данная плата обладает невысокой стоимостью, компактным корпусом, встроенным графическим ядром, аппаратной поддержкой видеокамеры. Как показали испытания, для поставленной задачи мощности этого одноплатного ПК хватает.

Базовая функциональная схема прибора

Функционирование разработанного прибора и алгоритма заключается в следующем процессе.

Объектив формирует на фотоприёмнике (ФП) изображение участка дороги. ФП превращает па- 
дающий поток излучения в электрический сигнал, который проходит усиление, аналого-цифровое преобразование, предварительную обработку, фильтрацию и записывается в буферное оперативное запоминающее устройство (ОЗУ), регистрируя информацию о разметке участка дороги и положение автомобиля относительно этой разметки. Дальнейшая обработка в процессоре исключает неинформативные участки, уменьшает шумы, выполняет фильтрацию изображения, детектирует разметку и положение транспортного средства и, в зависимости от дорожной ситуации, выдает предупреждение.

В состав детектора разметки входят (рис. 4):

- Объектив оптического устройства;

- Фоточувствительный прибор с переносом заряда (ФППЗ);

- Блок электронный;

- Программное обеспечение;

- Комплект монтажных частей.

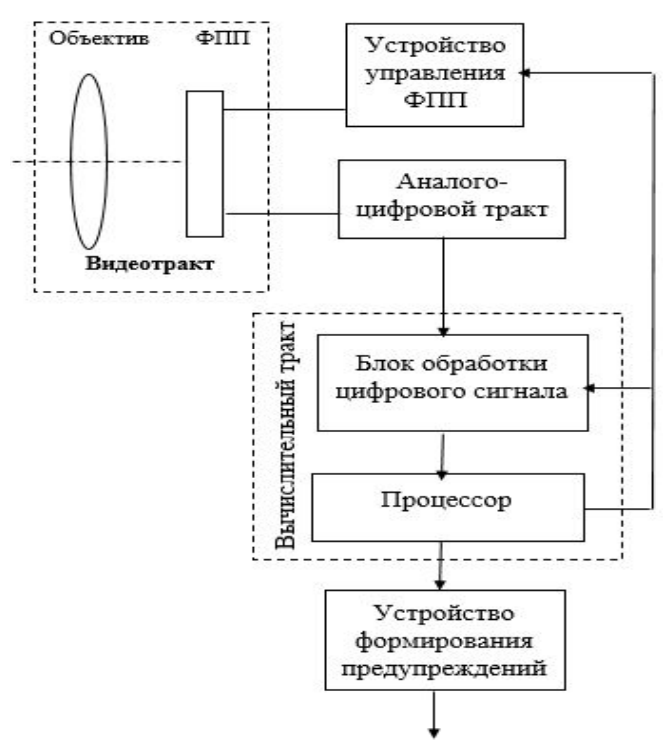

Рис. 4. Функциональная схема детектора дорожной разметки

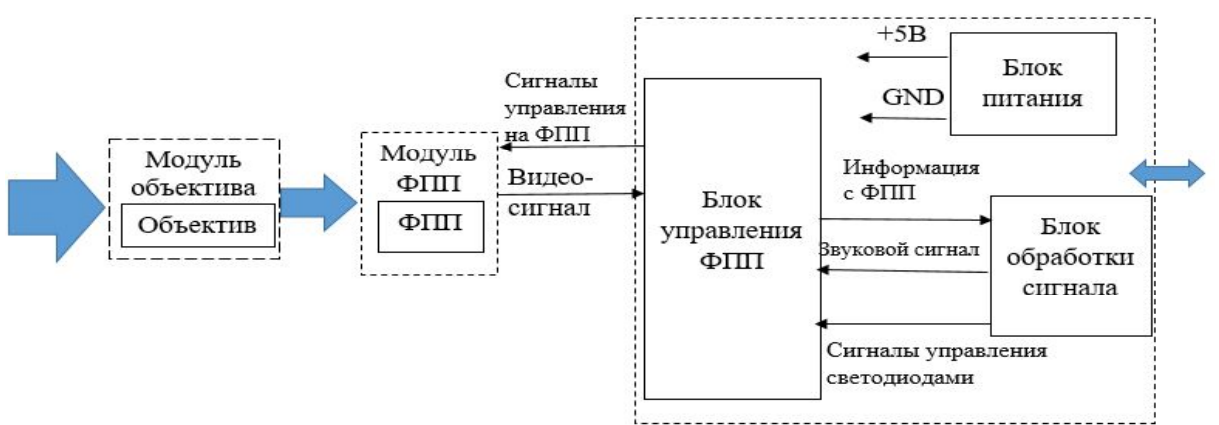

Рис. 5. Основные функциональные связи между модулями и электронными блоками

Модуль объектива включает в себя широкоугольный объектив, который способен обеспечить высокое качество изображения с минимальными геометрическими искажениями.

Прибор включает в себя несколько функциональных трактов, краткая характеристика которых приведена ниже (рис. 5):

- Видеотракт.

Содержит объектив и фотоприёмник. Видеотракт обеспечивает получение высококачественного изображения участка дороги.

- Аналого-цифровой тракт.

Состоит из видеоусилителя, аналогоцифрового преобразователя (АЦП), порогового устройства и буферного оперативного запоминающего устройства (ОЗУ). Тракт обеспечивает усиление аналогового видеосигнала с фотоприёмника, превращение в цифровой 12-разрядный код и первичную обработку видеоинформации с целью сжатия ее для дальнейшей вторичной обработки в вычислительном тракте.

- Вычислительный тракт.

Тракт обеспечивает обработку видеоинформации, управление всем вычислительным процессом, нахождение дорожной разметки и оценку положения автомобиля по отношению к разметке [10].

- Устройство формирования предупреждения. Управляется процессором. Обеспечивает формирование предупреждение для водителя.

Реализация алгоритма

Работа прибора построена на анализе изображения - компьютер ищет на снимке длинные вытянутые объекты (разметку) по пороговому эксцентриситету и помечает их (Рис. 6).

Разработка и отладка алгоритма осуществлялась в среде MatLab с применением Image Processing Toolbox и дальнейшим портированием в язык C. 
ISSN 0201-744X, ISSN 0321-2211

Методи і системи оптично-електронної та цифрової обробки сигналів

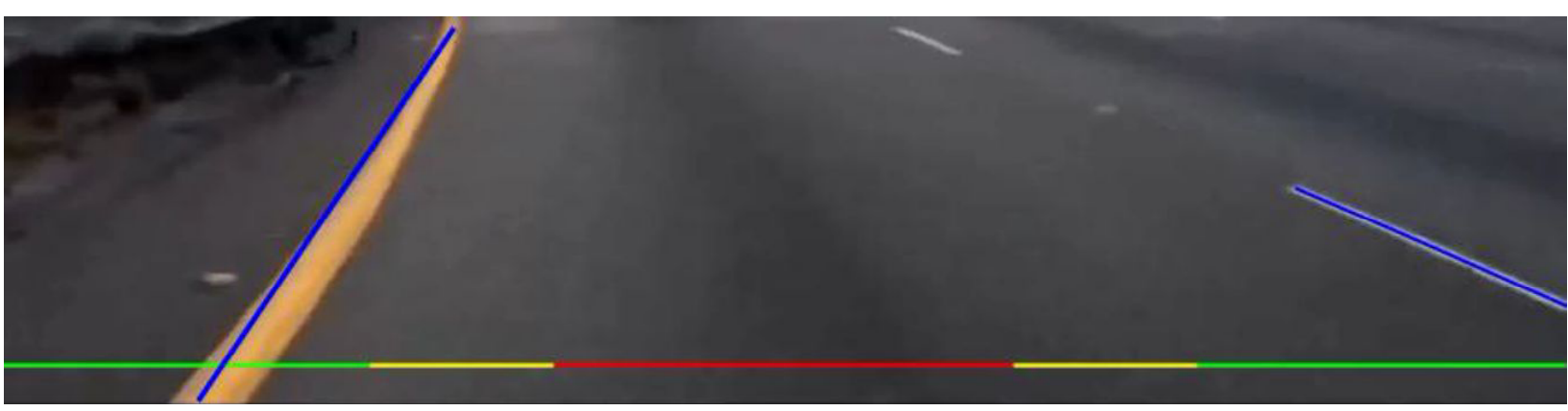

Рис. 6. Пример работы алгоритма нахождения дорожной разметки

\section{Выводы}

Анализ существующих систем активной безопасности выявил ряд бесспорных преимуществ «электронных ассистентов», а также доказал эффективность их применения. Однако был выявлен ряд недостатков, основным из которых является отсутствие универсальности, мобильности. Каждый автопроизводитель разрабатывает такие системы под свои нужды, не говоря об устаревших автомобилях. Также алгоритм работы данных систем не является доскональным - наблюдаются ложные срабатывания.

Системы активной безопасности - это качественный шаг к приближению автономного вождения и повышению безопасности на дорогах общего пользования. Их дальнейшее развитие является обязательным, поскольку они уже контролируют ситуацию на дороге и обеспечивают безопасное передвижение. Данные системы работают крайне незаметно для водителя, но вносят дополнительную ясность в вождении.

Автопроизводителям удалось разработать и внедрить качественные системы активной безопасности ADAS. Сейчас невозможно представить современное транспортное средство без «электронных ассистентов». На этом автомобильные гиганты не остановились и активно внедряют автономные транспортные средства. В данный момент времени Autonomous Driving системы находятся на 3 этапе автономности, предоставляющие автономность в предсказуемом трафике движения, но пока эти системы не способны взять на себя полную автономность вождения, особенно в сложных дорожных условиях.

Наиболее перспективной и безопасной считается Autonomous Driving Car, поскольку содержит все системы и датчики ADAS и упрощает процесс передвижения.

В результате исследования был предложен и разработан прибор - детектор полосы движения, который должен повысить безопасность дорожного движения. Данный детектор лишён недостатков рыночных решений, поскольку нет привязки под конкретный автомобиль. Дальнейшее развитие детектора - адаптация под автономный автомобиль и возможность управления автомобилем при помощи электроники.

\section{Литература}

1. C. Liu and T. Ye, Run-Off-Road Crashes: An OnScene Perspective (DOT HS 811 500), National Highway Traffic Safety Administration (2011) p.125-128.

2. C. F. Lin and A. G. Ulsoy, "Time to Lane Crossing Calculation and Characterization of Its Associated Uncertainty," ITS Journal, vol. 3, no. 2 (1996), 8598. DOI: $10.1080 / 10248079608903710$

3. http://systemsauto.ru/active/active.html

4. "US: Mobileye intros smartphone connected driver assistance (ADAS) technology". Telematics News. 2012-01-12. Retrieved 2012-01-12.

5. Thrun, Sebastian (2010). "Toward Robotic Cars". Communications of the ACM. 53 (4): 99-106. DOI:10.1145/1721654.1721679.

6. Davies, Alex. Google's Lawsuit Against Uber Revolves Around Frickin' Lasers, WIRED.

7. RT Staff. Google Cartographer SLAM Library Now Open-Source - Robotics Trends.

8. Autonomous Car Development Platform from NVIDIA DRIVE PX2

9. "U.S. DOT Proposes Rear View Visibility Rule to Protect Kids and the Elderly | National Highway Traffic Safety Administration (NHTSA)". Nhtsa.gov. 2010-12-03. Archived from the original on 2014-07-14. Retrieved 2014-07-15.

10. European Roadmap Smart Systems for Automated Driving, European Technology Platform on Smart Systems Integration (EPoSS), 2015.

11. "NHTSA Announces Final Rule Requiring Rear Visibility Technology | National Highway Traffic Safety Administration (NHTSA)". Nhtsa.gov. 201403-31. Retrieved 2014-07-15.

12. Lee E. S., Lee D. G., Lee S. H. Opto-mechanical design and analysis of the MSC on the KOMPSAT2 20th Congress of the International Commission for Optics. - Changchun, China, 2005. 
УДК 621: 681.7

В. С. Стаднічук, Д. Ю. Кондратенко

Національний технічний університет Украйни "Кийвський політехнічний інститут імені

Ігоря Сікорського”, Київ, Украӥна

ОПТИКО-ЕЛЕКТРОННИЙ ДЕТЕКТОР ШЛЯХОВОЇ РОЗМІТКИ

У даній роботі розглянуті і проаналізовані основні причини виникнення ДТП, концепції автономного водіння, існуючі конструкції оптико-електронних систем активної безпеки, допомоги водінню, запропоновано розробку власного універсального оптико-електронного приладу для всіх типів автомобілів. Основним недоліком таких систем $\epsilon$ відсутність мобільності. Аналіз існуючих систем активної безпеки виявив ряд безперечних переваг оптико-електронних систем, а також довів ефективність їх застосування.

Рішення проблеми розпізнавання і обробки зображень $\mathrm{i}$, як наслідок, запобігання аварійної ситуації $є$ досить важливим аспектом безпеки і контролю дорожньої ситуації. Системи активної безпеки дозволяють коригувати помилкові дії водія, уникати екстрених ситуацій, підвищити безпеку дорожнього руху. Актуальною, затребуваною і цікавою темою цього дослідження є аналіз і розробка власного універсального приладу «консультації» водія (ADAS) в питанні активної безпеки. Робота приладу побудована на аналізі зображення - комп'ютер шукає на знімку довгі витягнуті об'єкти (розмітку) по пороговому ексцентриситету і позначає їх. Об'єктив формує на фотоприймачі (ФП) зображення ділянки дороги. ФП перетворює падаючий потік випромінювання в електричний сигнал, який проходить посилення, аналого-цифрове перетворення, попередню обробку, фільтрацію і записується в буферний оперативний пристрій (ОЗУ), ресструючи інформацію про розмітку ділянки дороги і положення автомобіля щодо цієї розмітки.

Обробка в процесорі виключає неінформативні ділянки, зменшує шуми, виконує фільтрацію зображення, детектирует розмітку і положення транспортного засобу i, в залежності від дорожньої ситуації, видає попередження. Даний детектор позбавлений недоліків ринкових рішень, оскільки немає прив'язки під конкретний автомобіль. Подальший розвиток детектора - адаптація під автономний автомобіль і можливість керування автомобілем за допомогою електроніки.

Ключові слова: ADAS, Autonomous Driving Car, SLAM, LIDAR, SAE, автопілотовані автомобілі, відеокамера, стереозір, система активної безпеки.

\section{S. Stadnichuk, D. Y. Kondratenko \\ National Technical University of Ukraine "Igor Sikorsky Kyiv Polytechnic Institute”, Kyiv, Ukraine \\ OPTICAL-ELECTRONIC DETECTOR OF ROAD MARKING}

In this work, we examined and analyzed the existing designs of opto-electronic active safety systems, driving assistance, the main causes of the accident, the concept of autonomous driving, proposed the development of its own universal optoelectronic device for all types of cars. Analysis of statistical data showed that in most cases, the cause of an accident is the drivers' mistakes, and as a result, leaving their lane. Especially dangerous is the departure to the oncoming lane. In this regard, for 10 years now, developments have been carried out in the field of detection of road marking. Automobile manufacturers offer ready-made Embedded systems, built in behind the interior mirror of the rear view and connected to the on-board computer of the car, which carries out further control of the car in a critical situation. The main disadvantage of ready systems of this class is the lack of mobility and low photosensitivity. As a result of this work, a lane detector was developed and debugged. This device is universal (it can be used with any car), compact, lightweight, durable. Power consumption does not exceed 20 Watt. The detector installing on the windshield behind the rearview mirror with the help of a special lock. A number of shortcomings, such as a weak aperture of the lens, were also revealed. The value of the aperture is directly related to the minimum illumination of the road, which is accessible for registration in a visual way. In connection with this, a proprietary high-aperture lens was proposed and developed, ensuring correct operation of the device even in the dark time of the day and allowing observation at large distances. The analysis of existing active safety systems revealed a number of indisputable advantages of opto-electronic systems, and also proved the effectiveness of their application.

Based on the classification of ADAS systems, an analysis was made of their advantages and disadvantages.

Keywords: ADAS, Autonomous Driving Car, SLAM, LIDAR, SAE, autopilot cars, video camera, stereo vision, active safety system, image processing, optics.

Надійшла до редакиії 21 березня 2018 року

Речензовано 05 квітня 2018 року 